\title{
Téoros
}

Revue de recherche en tourisme

\section{Le parc du mont Mégantic : processus de création et impacts sur le développement régional}

\section{Roger Nadeau et Nil Longpré}

Volume 14, numéro 1, printemps 1995

Les parcs : des réseaux en mutation

URI : https://id.erudit.org/iderudit/1077049ar

DOI : https://doi.org/10.7202/1077049ar

Aller au sommaire du numéro

Éditeur(s)

Université du Québec à Montréal

ISSN

0712-8657 (imprimé)

1923-2705 (numérique)

Découvrir la revue

Citer cet article

Nadeau, R. \& Longpré, N. (1995). Le parc du mont Mégantic : processus de création et impacts sur le développement régional. Téoros, 14(1), 13-16.

https://doi.org/10.7202/1077049ar d'utilisation que vous pouvez consulter en ligne. 


\section{Le parc du mont Mégantic : processus de création et impacts sur le développement régional}

Roger Nadeau et Nil Longpré"

100 ans exactement après la création du premier parc au Québec, celui de la montagne Tremblante en 1895, suivie de prèspar celledu parcdes Laurentides, nous assistons aujourd'hui à la naissance du dernier-né, celui du mont Méganticen Estrie.

Cette création se situe dans le prolongement du dernier plan d'action du ministère du Loisir, de la Chasse et de la Pêche (MLCP) présenté à la fin de 1992 . Cet énonó́ de politique permet au MLCP de faire connaîtue ses intentions en regard du développement du réseau, lequel passe à la fois par la consolidation des seize parcs déjà existants (10 parcs de conservation et 6 pares de récréation, sans oublier le projet conjoint du parc marin sur le Saguenay) et par la création de nouveaux parcs. Le plan d'action prévoit que d'ici cinq ans, quatre nouveaux parcs seront créés au sud du $50^{\text {r }}$ parallèle, soit au mont Mégantic, ì la Réserve faunique de Plaisance (Outaouais), à la Rivière Vauréal (lle d'Anticosti) et au Massif du Mont Valin (Saguenay-Lac-StJean)(1). Si les 85 millions de dollars annoncés sur cinq ans pour la mise en place de ce plan d'action sont réellement injectés aux fins prévues, on aum sûrement fait un pas important.

Le mont Mégantic représentera la région naturelle dite odes montagnes frontaliereso une région bordée par trois états estaméricains. Le mont Mégantic est situé à une soixantaine de kilomètres de la région sherbrookoise qui compte 125000 habitants et à moins de deux heures des zones métropolitaines deMontréalet de Québec. Le mont Mégantic répond donc tout à fait aux critères de base conduisant à l'implantation d'un parc provincial.

Un processus qui peut être long... L'idée de mettreen valeur l'énorme potentiel du mont Mégantic a commencé à circuler au milieu des années 1960 à l'initia-

Monsieur Roger Nadeau est directeur du département de géographie et teledétection et professeur de tourisme à I'Universits de Sherbrooke. Monsieur Nil Longpré est commissaire pour la MAC du Granit a la Corporation Tourisme Region de Megantic.

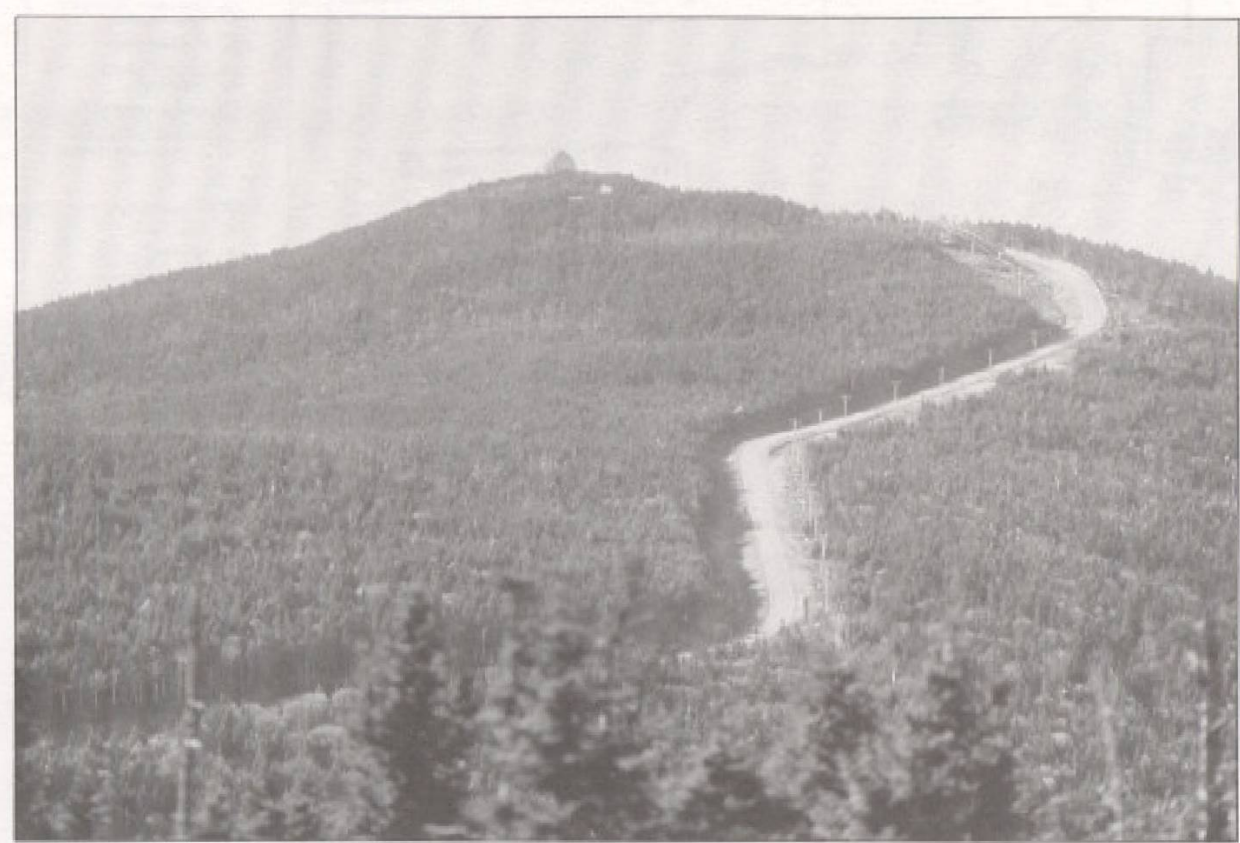

tive du Conseil régional de dúveloppement des Cantons de l'Est qui, dès ce moment, recommandait que cette zone soit vouée à la pratique des activités de plein air et préservée pour les générations futures. Et c'esten 1976 quele ministère du Tourisme signalait qu'il entendait intégrer le mont Mégantic dans son réseau de parcs provinciaux: ça fera 20 ans l'année prochaine!

L'avenir du massif s'est en partie joué en 1977 alorsqu'on ya implantél'observatoire astronomique le plus puissant de l'est de l'Amérique du Nord. Cet outil prestigieux de recherche va dorénavant marquer ala vocation scientifique de l'espace environnant à cause des exigences strictes liées au bon fonctionnement du télescope qui requiert une absence de pollution lumineuse. Cette implantation a augmenté considérablement la notoriété de la montagne auprès d'un grand nombre de personnes qui accentuèrent les pressions en faveurd'unemiseen valeur dusite, pressions qui furent canalisées par le Comité intermunicipal du mont $M e ́ g$ antic, au point qu'en 1983, les MRC du Haut SaintFrançois et du Granit protégeaient les terres de la montagne par règlement tandis que dès 1986, elles inscrivaient le projet de parc à leurs schémas d'aménagement respectifs $^{(2)}$.

En 1985, à l'occasion du premier Sommet socio-économique à se tenir en Estrie, l'ensemble des intervenants récréo-touristiques croyait bien que ça y était car le Gouvernement du Québec s'engageait à aller del'avant danscedossier avecdiligence. C'était sanscompter les subtilités politiques: en 1985, on assiste à un changement de gouvernement au Québec et en 1986, le nouveau ministre impose un moratoire sur la création de nouveaux parcs, moratoire qui s'éternisera jusqu'à l'automne 1991. Et c'est à la fin de l'été 1992, à l'occasion du dépôt du Plan d'action sur les parcs que le MLCP annonce enfin qu'on amorçait le processus de création du parc du mont Mégantic, le premier de cette nouvelle génération(?).

Près de 70 mémoires ont été présentés au Ministère lors des audiences publiques de 1993, lesquels permettaient à toutes les personnes concernées de réagir au plan préliminaire d'aménagement présentế par le MI.CPet d'indiquer clairementl'intérêt de la région à la réalisation de ce dossier majeur. Finalement, à la fin de l'été de 


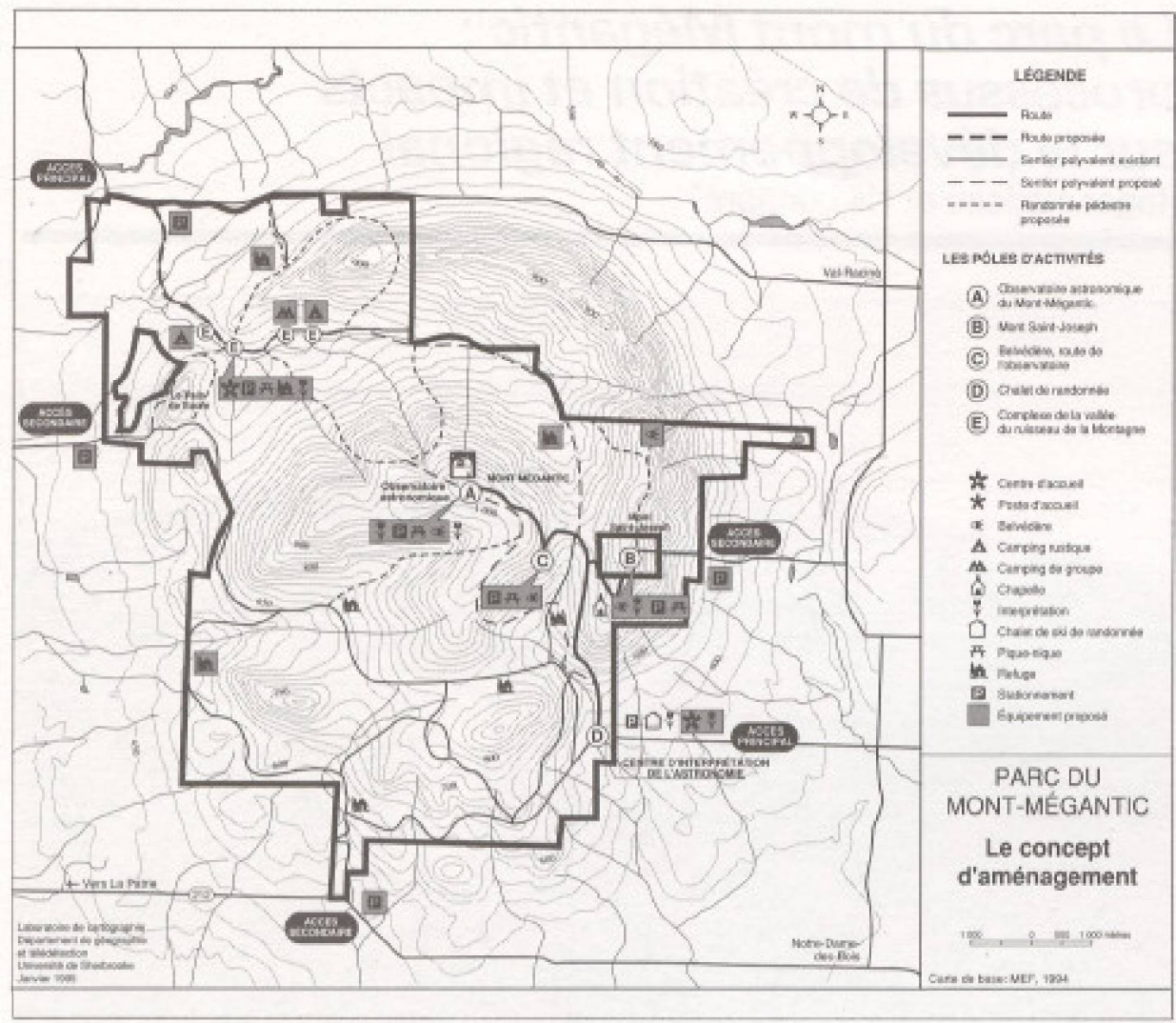

1994, le ministre Pierre Paradis a inauguré officiellement le Parc de conservation du Mont Mégantic. A son tour, le gouvernement qu*il représentait a été défait mais le point de non retour a été atteint et le plan final d'aménagement devrait être déposé au printemps de 1995. Le MI.CP est disparu: place au ministêre de l'Environnement et de la Faune!

C'est pourquoi, en éditorial, La Tribune de Sherbrooke saluait la conclusion de ce projet de la façon suivante: «L'incroyable patience de la communauté ceinturant le mont Mégantic est enfin récompensée: ce joyau de la nature estrienne accede au titre deparcprovincial. La nouvelle est d'autant plus réjouissante qu'il y a longtemps dans ce coin de pays qu'un tel projet rallie l'ensemble des citoyens: ${ }^{4 !}$

Pour compléter la description de ce processus, il faut signaler deux situations particulières. La première, c'est la qualitétrès professionnelle du travail de rechercheterrain effectuépar $l s$ gen $d u$ MLCP quiont passé à la loupe toutes les coutures de la montagne avant de présenter un plan provisoire d'aménagement et qui sont toujours à l'oeuvre pour élaborer le plan d'interprétation et la mise en place des infras- tructures. La seconde, c'est la place importante qui est laissée aux intervenants du milieu dans la gestion du parc via une table d'barmonaation qui donne des responsabjlités explicites aux gens du milieu sur les orientations de développement et sur le devenir du parc.

La route a été longue mais le Paredu Mont Mégantic est maintenant une réalité. Ce rerritoire de près de 60 kilomètres carrés s'élève jusqu" 1130 mètres d'altitude. Quant à l'Observatoire astronomique du Québec, il se situe à une altrtude de 1105 mètreset est accessible par un réseau routier tout à fait adéquat. Voilà deux spécificités de taillequi jouent en faveur du parcauprès des visiteurs aे venir.

Le plan d'aménagement prévoit que le territoire du parcsera divisé en trois parties selon un modèle de zonage qui a fait consensus, aLes zones de préservation s'étendent sur $32 \%$ de la superficie du parc, soit $18,6 \mathrm{~km}^{2}$. Des sentiers aménagés donnent accessà ceszones. Des critères tels que la valeur éducative importante, la fragilité du milieu, le potentiel floristique élevé et la représentativité permettent de délimiter ces espaces de conservation. La zone dianbiance couvte $67 \%$ de la superfi- cie du parc, soit $39,7 \mathrm{~km}^{2}$. Les secteurs de cette zone possèdent un potentiel pour la découverte du milieu et une capacité de support. Is offrent aussi la possibilité d'implanter des activités récréatives extensives. Pour leur part, les zones de services représentent $1 \%$ de la superficie du parc, soit $0,5 \mathrm{~km}^{2}$. L'observatoire astronomique, le chalet de randonnée et le poste d'accueil de la Vallée du ruisseau de la montagne font partie de ces zones destinées à l'accueil et à l'hébergement du public visiteurs (5).

Le potentiel pour l'interprétation est ici quasi illimité. On pense surtout aux domaines de l'astronomie, de la géologie, de la géomorphologie, de l'hydrographie et de la climatologie. La végétation et la faune, sans oublier le milieu humain, seront aussi omniprésent. C'est à partir de ces champs d'activités que le caractère «scientifiques du parc peut se démarquer pour en faire un produit d'appel wnique.

Il semble bien, a priori, que ce produit d'appel fort aura comme déclencheur l'observatoire astronomique et le Centre d'interprétation astronomigue $d u$ Mont Mégantic. Il nous apparaît en effet quesans cette dernière composante du projet, l'impact récréo-touristique serait beaucoup moindre. C'est pourquoi, à l'oocasion des audiences publiques, la très forte majorité des mémoires ont demandé avec insistance au MLCP de faire une place nettement plus importante au centre d'interprétation pour en faire une pièce maîtresse de ce dossier. Pour mieux envisager les perspectives touristiques associées a ce type d'infrastructure, on n'a qu'à rappeler que depuis 1982, près de 275000 visiteurs ont été attirés par l'observatoire, même s'il n'y avaitsur placeaucun service d'accueil, aucun service d'hygiène, aucun casse-croutte, bref... rien! En 1986, le Groupe conseil Coopers \& Lybrand avait pourtant déposé une étude de faisabilité sur ce centre d'interprétation. On pourra d'ailleurs s'y référer car il semble bien que c'est le concept développé par les consultants qui sera la source principale d'inspiration ${ }^{(6)}$.

Ce volet touristique est fort important car si la population régionale se réjouit quel'on ait enfin reconnu les grandes ressources de cet espace en le sacrant parc de conservation, elle souhaite aussi vivement qu'il en découle des retombées économiques notables. Bien des intervenants, en effet, voient dans le complexe Parc/Centre d'interprétation/Observatoire, le spoumon-moteurs 
et la pierre angulaire d'une station touristique majeure en éclosion ${ }^{(7)}$.

Nous verrons donc ici dans quel contexte ces dossiers majeurs arrivent en région et les impacts qu'ils sont susceptibles de provoquer sur l'industrie touristique.

\section{La région de Mégantic,} nouvelle destination touristique Il faut savoir que déjà la MRC du Granita, au cours des quatre dernières années, mis l'accent sur le développement de son offre récréo-touristique. Il faut dire aussi au départ que cette région est choyée sur le plan des richesses naturelles puisqu'on y relève trois grands lacs auxquels s'ajoutent de nombreux plans d'eau secondaires, des montagnes de plus de 1000 mètres et des collines à perte de vue ainsi que de vastes étendues forestières qui agrémentent les paysages.

C'est dans ce riche enviromement que quinze nouvelles entreprises touristiques se sont implantées depuis le printemps 1991. De ce nombre, huit entreprises oeuvrent dans le secteur del'hôttellerie tout en offrant, pour la plupart, des activités récréatives directement accessibles sur le site. Ces entreprises surtout constituées d'auberges et de gites touristiques ont permis d'ajouter 50 chambres au parc hôtelier de la région méganticoise pour un total, à ce jour, de 270 chambres.

L'offre hôtelière est, comme on peut le constater, plutôt limitée. Fort heureusement, on peut compter sur quelques campings bien aménagés dont celui du Club Vacances Baie des Sables localisé en bordure du lac Mégantic et sur plusieurs dizaines de résidences secondaires mises en location chaque été.

La création du Pare du Mont Mégantic apportera un second souffle au développement de la région de Mégantic. On peut effectivements'attendreà ce que la mise en valeur de ce massif montagneux, un des joyauxdenotrepatrimoine naturel, entraîne un impact considérable sur l'affluence touristique d'une part et le développement de nouvelles entreprises touristiques, d'autre part. Les lignes qui suivent en font la démonstration.

\section{Une clientèle branchée sur la nature}

L'identification des éléments motivant notre clientèle à venir séjourner dans la région pendant leurs vacances nous éclaire sur le pouvoir attractif de notre milieu naturel. Ainsi, dans notre étude de la clientèle touristique, on constate que la beauté des paysages et la nature sont les éléments qui agissent le plus comme déclencheur auprès de notre clientèle $31 \%$ des réponses). Le mont Mégantic luimême avec son observatoire (17\%) est le deuxième élément en importance suivi des activités de plein air et du camping (13 $\%)^{(i)}$.

Par ailleurs, lorsqu'on examine les principales forces de la région de Mégantic identifiées par les touristes, on apprend que les éléments associés directement à notre environnement tels la beauté des paysages, le lac Mégantic et le mont Mégantic, la tranquillité et le calme totalisent $85 \%$ des réponses: ${ }^{(0)}$.

Face a ces constats, il y a tout lieu de croire que la mise en place du Pare du Mont Mégantic, avec tout ce que cela suppose en terme d'infrastructures et d'équipements récréo-touristiques, aura un effet structurant sur le produit nature de la région méganticoise. Dans la mesureoù on assure au minimum la promotion de ce nouveau parc, on doit donc s'attendre à une augmentation de l'achalandage touristique.

\section{Impact sur le développement,} I'exemple du Parc de Frontenac

Le Pare de Frontenac est un parc provincial de conservation. Il possede ainsi le mêmestatutquele ParcduMontMégantic. Ce parc, localisé dans la portion nordouest de la région de Mégantic, touche cinq municipalités du territoire de la MRC qui ont été regroupées sous l'appellation du secteur du Parc de Frontenac. La partie norddu parc débordedela MRCdu Granit et se retrouve dans la MRC de l'Amiante. Créé en 1987, le parc a au fil des ans fait l'objet de plusieurs amémagements récréotouristiques bien qu'il nesoit soit développé qu'à $65 \%$ de son potentiel.

Nous avons, précédemment, fait état que 15 entreprises touristiques ont démarré leur opération depuis 1991 dans la région de Mégantic. Il est extrêmement intéressant de souligner que de ce nombre, 10 entreprises regroupant près de $70 \%$ des investissements engendrés sont du secteur du Pare de Frontenac.

Pour avoir été associé de très près dans le montagne des plans d'affaires de chacune de ces 10 entreprises, nous pouvons affirmer que la présence du parc dans le voisinage immédiat des entreprises a été un élément déterminant permettant de justifier la fréquentation prévisionnelle de ces entreprises et conséquemment de faire la démonstration de leur rentabilité. D'ailleurs, certaines de ces entreprises ont un lien direct avecleparcà tel point qu'elles n'existeraient pas si le parc lui-même n'existait pas.

Nous nous devons cependant de préciser que la seule présence du parc n'explique pas tout. Les intervenants familiers avec la dynamique du développement régional savent bien qu'on peut rarement attribuer à un seul facteur le succès d'une région ou d'un secteur donné.

Nous sommes toutefois convaincus que l'implantation du Parc du Mont Mégantic aura un effet d'entraînement remarquable sur le développement de petites entreprises touristiques tout autour du massif montagneux. Conséquemment, on peut donc s'attendreà ce que des établissements hôteliers assortis de petites auberges et de gites touristiques ouvrent leurs portes d'ici les cinq prochaines années. Il en ira de mêmepour un certain nombre d'entreprises offrant des services et des attraits récréotouristiques.

En fait, nous anticipons un développement encore plus important dans la périphérie du Parc du Mont Mégantic que ce qu'on a connu au Parc de Frontenac. Et ce développementsera sansaucun doute beaucoup plus rapide. Deux raisons bien précises nous confortent dans ce raisonnement.

\section{Le mont Mégantic et l'astronomie,} une notoriété déjà bien établie

Comme nous l'avons mentionné précédemment, environ 275000 personnes sont passées à l'observatoire astronomique depuis 1982. Ainsi, au fil des ans, la région de Mégantic a progressivement été associée à l'Observatoire du Mont Mégantic. Il ne fait nul doute actuellement qu'il s'agit du site touristique quidisposede la plusgrande notoriéré de tout le territoire de la MRC. Nous avons à chaque année l'occasion de vérifier cet état de fait lors de notre participation à des salons promotionnels du genre des Salons vacances et loisirs d'été de Montréal et de Québec. En nous apercevant sous la bannière «région de Mégantio , les visiteurs des Salons nous associent, dans une très forte proportion, à l'observatoire. 
En fait, l'observatoire est vite devenu et est encore aujourd'hui l'attrait touristique numéro 1 des MRCdu HautSaint-François et du Granit.

Cette notoriété n'a pas été sans poser des problèmes et des soucis aux dirigeants de l'observatoire. Car, comme nous l'avons précisé auparavant, l'observatoire a été aménagé et construit à des fins de recherche scientifique et non à des fins d'accueil touristique. C'est cettesituation qui a forcé lemilieuà réagir età envisager la possibilité de bâtir un Centre d'interprétation de l'astronomie (CIA).

Cela nous amèneà la deuxième raison nous permettant d'anticiper un développement important dans le secteur du Parc du Mont Mégantic.

\section{Le Centre d'interprétation de l'astronomie, produit d'appel par excellence}

Il y a longtemps qu'on parle de l'implantation d'un tel centre au mont Mégantic; certains vous diront 10 ans, d'autres 15 ans... Bref, l'idée n'est pas nouvelle. Ce qui est nouveau, c'est que le financement nécessaire la la matérialisation de ce projet de 2,4 millions de dollars est à toute fin pratique réglể $(10)$.

Cela dit, selon l'échéancier actuel de la Société de développement touristique du Mont Mégantic, promoteur du projet, on verra l'ouverture du centre en juin 1996. Quatre modules distincts composeront le Centre d'interprétation de l'astronomie: le moduleaccueil, le module interaction, le module simulation ainsi que le module observation. Enfin, les très nombreux visiteurs allant déjà à l'observatoire auront à leur disposition un bâtiment axé sur l'astronomie qui leur est destiné et qui dispose de tous les services indispensables à une visite agréable.

Le Centre d'interprétation de l'astronomie sera, sans conteste, l'élément clé du Parc du Mont Mégantic. La thématique

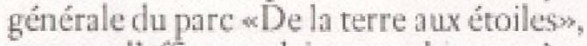
permet d'affirmer clairement le caractère distinctifdu Parcdu Mont Méganticparmi les autres parcs provinciaux ${ }^{(11)}$.

Pour la région de Mégantic, le Centre d'interprétation de l'astronomie sera le produit d'appel par excellence de notre industrie touristique. Il n'est donc pas surprenant que la réalisation de ce centre ait été retenu comme enjeu stratégique prioritaire par Tourem en $1993^{(12)}$. II n'est par surprenant également que dans le Plan de développement de l'Association touristique de l'Estric, on ait identifié ce projet parmi une liste de 7 projets moteurs à développer sur le territoire estrien ${ }^{(13)}$. Il est finalement remarquable de relever dans le Plan de développement stratégique du Conseil régional de développement de l'Estrie, une mention indiquant, en regard aux attraits touristiques, qu'il faut soutenir le développement d'attraits majeurs à effets structurants, notamment celui du mont Mégantic s(14). A notre connaissance, il s'agit lì du seul projet nommément identifié dans cette abibles du développement de l'Estrie s'appliquant de 1993 à 1998. Par ailleurs, l'aménagement du CIA à l'intérieur du Parccadre on ne peut mieux avecl'espritdes produits touristiques alliant la culture et la nature ${ }^{(15)}$.

La fréquentation anticipée au CIA pour la première année d'opération est de 42000 visiteurs. Cela nous apparật plutôt conservateuret nousprévoyonsun achalandage beaucoup plus substantiel dès la deuxième année d'ouverture. Cette situation éventuelle sera encore plus probable si la tendance actuellese maintient dans l'industrie touristique québécoise ${ }^{(16)}$.

Tout comme l'achalandage naturel de l'observatoirea amené la création du CIA, l'achalandagedu CIA suscitera l'avènement deplusieurs autresentreprises touristiques. Voilà pourquoi on parle ici d'un projet à effets structurants et pourquoi nous sommes convaincus que le développement touristique se fera encore plus rapidement dans la périphérie du Parc du Mont Mégantic que ce qui a été connu dans la périphérie du Parc de Frontenac.

\section{Conclusion}

La miseen place du Parcdu $M$ ont Mégantic etla concrétisation du Centred'interprétation de l'astronomie viennent de jeter les bases pour ancrer la croissance de l'industrie touristique de la région de Mégantic.

La créntion de ce deuxième parc permet de fairedela MRCdu Granit la seule MRCdu Québec, sur les 96 exastantes, à disposer de deux parcs provinciaux surson territoire. $\mathrm{A}$ cela s'ajoute, à mi-chemin entre les deux, le Club Vacances Baie des Sables de Lac Mégantic aractérisé par sa vocantion nautique et son camping 4 étoiles. A eux trois, ces sites forment ce qu'on peut appeler le triangle du plein air. Le Parc du Mont Méganticamène ainsi la confirmation dela vocation écotouristique de la région méganticoise.

Le défi est maintenant double. Il consiste, d'une part, à définir une stratégie de promotion et de commercialisation en collaboration avec les intervenants touristiques régionaux. Cela demandera du temps bien sûr, mais devrait être relativement aisé. D'autre part, il nous faut maintenir le cap sur le développement écotouristique des potentiels de la région. Il s'agit là, à notre humbleavis, d'un défi detaille qu'on pourra relever seulement si les autorités du milieu (municipales et autres) sont conscientes des enjeux et sont ouvertes aux propos de quelques visionnaires qui n'ont pas toujours tout le poids nécessaire. Beaucoup d'énergies seront doncdéployées carl'enjeu en vaut largement la peine. $f$

\section{NOTES}

(1) MLCP. La nature en héritage, Plan d'action sur les parcs, Québec, Gouvernement du Ouébec, 1992, p. 14.

(2) MLCP, De la terre aux b́tolles - Parc du Mont Mégantic, Québec, Gouvernement du Québec, 1993 , pp. 7-8.

(3) Cabinet du Ministre, Notes pour allocution du ministre du Loisir, de la Chasse et de la Péche. monsieur Gaston Blackburn, a l'cccasion de la conference de presse concernant le futur parc du Mont Megantic, La Patrie, 1992, pp. 34.4.

(4) François Gougeon. Un eare a protéger, La Tri bune, Sherbrooke, 18 aoot 1992.

(5) Marie-Andrée Piédalue, Plan d'interprótation Parc de Mont Mógantic - De la terre aux étoiles, Québec, ministèredel'Environnement et de la Faune, Diection du plein air et des parcs, $1994, \mathrm{pp}, 9-10$

(6) Coopers \& Lybrand, Étude de faisabilite duun centre d'interprétation de la nature cosmique (Mont Mégantic). Montréal, ministère du Tourisme, 1986,85 pages + annexes.

(7) R. Nadeau et Alain Dion, Le parc national scientifique du mont Mégantic, mémoire présentéau ministere du Loisir, de la Chasse et de la Pêche dans le cadre des audiences publiques entourant la création du Parc du Mont Mégantic, Sherbrooke, 1993,32 pages.

(B) Claude $\downarrow$. Bernard, Profil de la clientèle touristique de la région du Lac Mégantic, rapport de baccalaurbat, Université de Sherbrooke, 1992, p. 37. Préalablement à la rédaction de ce rapport une enquete auprès de 385 touristes a été réalisée par une equipe de 6 étudiants dirigés par monsieur Roger Nadeau de I'Université de Sherbrooke.

(9) Idem, p. 32

(10) Un protocole d'entente reste à signer awec lo BFDRIO) alors qu'on attend la réponse du CRD Estrie en avril 1995 pour une contribution tinan. cière complémentaire.

(11) MEF, Plan d'interprétation - Parc du Mont Mégantic - De la terre aux étoiles, 1994, p. 55

(12) Tourem, Plan stratogique de développement de rindustrio touristique de la région de Mégantic, 1993-1996, 1993, p. 78.

(13) ATE, Plan de développement touristique do TEstrie, rapport final Sherbrooke, 1988, p. 80.

(14) CRDE, Plandedávaloppement de rEstrie, 1993. 1998. 1993, p. 111.

(15) Ministère du Tourisme, Énoncé de politique et plan d'action en matidre de tourisme, Quebec, $1992,95 \mathrm{p}$.

(16) Les Affaires. Le tourisme en 1995: une autre bonne saisan s'annonce, samedi, 17 décembri $1994, p, 16$ 